\title{
Metformin restores electrophysiology of small conductance calcium-activated potassium channels in the atrium of GK diabetic rats
}

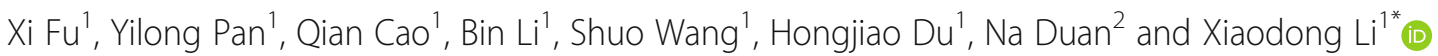

\begin{abstract}
Background: Small conductance calcium-activated potassium channels (SK channels) play a critical role in action potential repolarization in cardiomyocytes. Recently, the potential anti-arrhythmic effect of metformin in diabetic patients has been recognized, yet the underlying mechanism remains elusive.

Methods: Diabetic Goto-Kakizaki (GK) rats were untreated or treated with metformin (300 mg/kg/day) for 12 weeks, and age-matched Wistar rats were used as control ( $n=6$ per group). Electrocardiography, Hematoxylin-eosin staining and Masson's trichome staining were performed to assess cardiac function, histology and fibrosis. The expression levels of the SK channels in the myocardium were determined by real-time PCR and Western blotting. The electrophysiology of the SK channels in the cardiomyocytes isolated from the three groups of rats was examined by patch clamp assay, with specific blockade of the SK channels with apamin.

Results: Metformin treatment significantly reduced cardiac fibrosis and alleviated arrhythmia in the diabetic rats. In the atrial myocytes from control, GK and metformin-treated GK rats, the expression of KCa2.2 (SK2 channel) was down-regulated and the expression of KCa2.3 (SK3 channel) was up-regulated in the atrium of GK rats as compared with that of control rats, and metformin reversed diabetes-induced alterations in atrial SK channel expression. Moreover, patch clamp assay revealed that the SK current was markedly reduced and the action potential duration was prolonged in GK atrial myocytes, and the SK channel function was partially restored in the atrial myocytes from metformin-treated GK rats.
\end{abstract}

Conclusions: Our data suggests an involvement of the SK channels in the development of arrhythmia under diabetic conditions, and supports a potential beneficial effect of metformin on atrial electrophysiology.

Keywords: Small conductance calcium-activated potassium channel, SK channel, Metformin, Diabetes, Arrhythmia

\section{Background}

According to International Diabetes Federation, the global prevalence of diabetes was 415 million in 2015 and the number is estimated to be 642 million by 2040 [1]. Type 2 diabetes mellitus (T2DM) accounts for $90-95 \%$ of all diagnosed cases of diabetes, and it also confers an approximately twofold-increased risk of cardiovascular diseases $[2,3]$. In addition to the well-documented coronary artery disease and related cardiac events, cardiac electrical

\footnotetext{
* Correspondence: Ixd1894021@126.com

'Department of Cardiology, Shengjing Hospital of China Medical University, Shenyang 110004, People's Republic of China

Full list of author information is available at the end of the article
}

disturbance are recognized as critical cardiovascular complications of T2DM and may lead to tachycardia, fibrillation, cardiomyopathy, and even death [4-6]. However, the mechanisms underlying the relation between T2DM and arrhythmia is not fully understood.

Small conductance calcium-activated potassium channels (SK channels) are important players in cardiac action potential repolarization [7]. The SK channels consists of three isoforms, namely, KCa2.1, KCa2.2 and KCa2.3, which are encoded by KCNN1, KCNN2 and KCNN3, respectively. All the three SK isoforms are detected in rat atrial and ventricular myocytes with higher abundance of SK1 and SK2 in the atria than that in the ventricles [8]. A

(c) The Author(s). 2018 Open Access This article is distributed under the terms of the Creative Commons Attribution 4.0 International License (http://creativecommons.org/licenses/by/4.0/), which permits unrestricted use, distribution, and reproduction in any medium, provided you give appropriate credit to the original author(s) and the source, provide a link to the Creative Commons license, and indicate if changes were made. The Creative Commons Public Domain Dedication waiver (http://creativecommons.org/publicdomain/zero/1.0/) applies to the data made available in this article, unless otherwise stated. 
genome-wide study has identified common genetic variants of $K C N N 3$ that were associated with lone atrial fibrillation [9]. In addition, a selective SK blocker has been shown to significantly prolong atrial refractoriness and reduce atrial fibrillation duration in a canine model of atrial fibrillation [10]. These findings imply an important role of the SK channels in the maintenance of atrial electrophysiology. However, whether the SK channels are involved in T2DM-associated arrhythmia remains to be elucidated.

Metformin is currently considered one of the first-line treatments for T2DM [11]. Recently, the anti-arrhythmic effect of metformin has been gradually recognized. In a 13 -year dynamic cohort study of 645,710 patients with T2DM, metformin treatment was associated with a significantly reduced incidence of new-onset atrial fibrillation [12]. It remains unknown whether metformin exerts a direct corrective effect on the electrical activity in the diabetic atrial myocytes. Hence, the current study aims to explore the mechanism underlying the antiarrhythmic effect of metformin in a T2DM rat model with a special focus on the SK channels.

\section{Methods}

\section{Animals}

Twelve male Goto-Kakizaki (GK) T2DM model rats (10 weeks old, $249.83 \pm 4.68 \mathrm{~g}$ ) were allowed to access to standard chow and water ad libitum and maintained in a controlled environment $\left(22 \pm 2{ }^{\circ} \mathrm{C}\right.$, relative humidity of $55 \pm 5 \%$ and 12,12-h light-dark cycle). The rats' body weights and blood glucose levels (determined by an Accu-Chek glucometer, Roche) were measured three times a week. Age-matched, male, non-diabetic Wistar rats $(249.50 \pm 4.48 \mathrm{~g})$ were used as the control (Con). After two-week adaptation, fasting blood glucose and blood insulin levels were measured and an intraperitoneal glucose tolerance test was performed [13] to conform the onset of diabetes in the GK rats. GK rats in the metformin-treated group (Met) $(n=6)$ received intragastric administration of metformin ( $300 \mathrm{mg} / \mathrm{kg} /$ day) since 12 weeks of age for three months, while an equal volume of citric acid buffer was intragastrically administrated daily to the rats in the GK and Con groups $(n=6$ each group). This high dose of metformin was chosen because that long-term treatment with high-dose metformin could alleviate vascular dysfunction and rescue SK channel-mediated vasodilation in diabetic rats $[14,15]$. At the end of three-month metformin treatment, electrocardiogram (ECG) was performed on all rats to monitor the cardiac electrical activity.

\section{Hematoxylin-eosin and Masson's trichome staining}

After the rats were euthanized by i.p. injection with $100 \mathrm{mg} / \mathrm{kg}$ pentobarbital sodium, the atrium were excised along with the outline of the atrial appendages, fixed in
4\% paraformaldehyde and sectioned. Hematoxylin-eosin and Masson's staining were performed as previously described [16]. The images of stained atrial sections were captured under a light microscope, and the proportion of fibrotic areas was determined using the NIS-Elements F3. 0 software (Nikon, Tokyo, Japan).

\section{Western blot}

To assess the expression levels of SK channels in the atrium, western blotting was conducted according to a previously described method [17]. The primary antibodies used here included anti-KCa2.1 (1:400; Alomone, Israel), anti-KCa2.2 (1:200; Millipore, USA), anti-KCa2.3 (1:500; Abcam, UK), and anti-GAPDH (1,2000; Santa Cruz, USA). GAPDH served as the loading control. ImageJ software (NIH, Bethesda, MD) was used to analyze the intensity of the target bands.

\section{Real-time PCR}

Total RNAs in rat atrial tissues were extracted using an RNeasy kit (Qiagen, CA), and reversely transcribed into cDNA using a PrimeScript RT Reagent Kit with gDNA Eraser (Takara) according to the manufacturer's instructions. The sequences of the sense (5'-3') and antisense (5'-3') primers are as follows: rKCNN1: CCTTCCTGT CCATCGGCTAC, GCGTTTTTAACCCGCTTGGT; rK CNN2: ACTAGCAACTTCCTTGGAGCA, GCAACCT GCACCCATTATTCC; rKCNN3: CACCTTCCCCAA AGCCAACA, CGATCACAAAGAGCTGTACTTCC; rG APDH: GGCAAGTTCAACGGCACAGT, TGGTGAA GACGCCAGTAGACTC.

\section{Isolation of rat atrial myocytes and patch-clamp assay}

Rat atrial myocytes was isolated as previously described using the Langendorff apparatus [18]. The velocity of heart retrograde perfusion was $1 \mathrm{ml} / \mathrm{min}$. SK currents were apamin sensitive. SK currents = baseline $\mathrm{K}^{+}$current before apamin - $\mathrm{K}^{+}$current after $100 \mathrm{pM}$ apamin (a specific SK channel blocker). According to a standard method [19], whole-cell $\mathrm{K}^{+}$currents in rat atrial myocytes were detected using an EPC10 amplifier, wherein all signals were acquired at $5 \mathrm{kHz}$ and analyzed by Patchmaster software (version 2.72, HEKA Electronics, Lambrecht/Pfalz, Germany).

Quiescent, calcium-tolerant, spindle-shaped cells with clear cross striation were used for action potential recordings at $35{ }^{\circ} \mathrm{C}$. Action potential was elicited as described previously [19]. At the moment of 50 and $90 \%$ repolarization, the action potential duration (APD) was detected and recorded as $A P D_{50}$ and $A P D_{90}$, respectively. In addition, resting potential (RP), action potential amplitude (APA), $\mathrm{APD}_{50}$ and $\mathrm{APD}_{90}$ were recorded before and after exposure to $100 \mathrm{pM}$ apamin for $15 \mathrm{~min}$. The action potential recordings without apamin exposure 
showed the normal state, and the results after apamin exposure demonstrated the condition of SK channel blockade.

\section{Statistical analysis}

The data are expressed as mean \pm SEM. Control and GK groups were compared using $t$ test. For multiple comparisons, one-way analysis of variance (ANOVA) followed by Fisher's least significant difference test was used when the data conformed Gaussian distribution and homogeneity of variance, and nonparametric Kruskal-Wallis test with Dunn's multiple comparisons was used otherwise. Statistical analyses were performed using the SPSS software (version 19.0, Chicago, IL) and GraphPad Prism (version 6.0, La Jolla, CA).

\section{Results \\ Metformin alleviated structural remodeling and arrhythmia in the atrium of GK rat}

GK rats, as a T2DM model, displayed hyperglycemia (Table 1) and glucose intolerance (Fig. 1a; Additional file 1: Table S1) by 12 weeks of age, confirming of the diabetic state at the beginning of metformin treatment. After 12-week treatment, the anti-diabetic metformin significantly increased fasting blood glucose and in GK rats, but it also decreased rat body weight (Table 2).

Hematoxylin-eosin staining showed that the atrial myocytes in GK rats were disordered and distorted with unevenly stained cytoplasm and irregular nuclei. By contrast, GK rats with metformin treatment showed a less degree of atrial myofilament irregularity (Fig. 1b). Moreover, Masson's staining revealed that the GK rats developed interstitial fibrosis, and metformin attenuated atrial fibrosis in GK rats (Fig. 1c and d, $P<0.01$; Additional file 1: Table S2). In the meanwhile, ECG of GK rats showed accelerated heart rate, junctional rhythm, separation of $P$ and QRS waves, irregular $P$ waves, and partial blockade of electrical conduction (Fig. 2). In contrast, ECG of metformin-treated GK rats showed sinus rhythm and relatively regular $P$ waves (Fig. 2). These results demonstrated a protective effect of metformin on the structure and electrophysiology of the atrium under diabetic conditions.

Table 1 Diabetic conditions of 12-week old GK rats before metformin treatment

\begin{tabular}{lll}
\hline & Con & GK \\
\hline Weight $(\mathrm{g})$ & $249.50 \pm 4.48$ & $249.83 \pm 4.68$ \\
FBG $(\mathrm{mM})$ & $4.38 \pm 0.24$ & $12.37 \pm 0.23^{* *}$ \\
FINS (mIU/l) & $20.46 \pm 1.11$ & $27.62 \pm 2.53^{*}$ \\
\hline
\end{tabular}

FBG fasting blood glucose, FIN fasting insulin, $n=12$ per group

${ }^{*} P<0.05$ vs Con, ${ }^{* *} P<0.01$ vs Con

\section{Metformin modulated the expression of SK channels in} the atrium of GK rat

To investigate the mechanisms underlying diabetic arrhythmia and the anti-arrhythmic effect of metformin, the expressions of the SK channels in the atria of GK rats with and without metformin treatment were examined. Western blot results showed that the level of KCa2.2 in GK atrium was approximately half of that in control atrium and the level of $\mathrm{KCa} 2.3$ in GK atrium was $1.98 \pm 0$. 5 fold of that in control atrium (Fig. 3a and b; Additional file 1: Table S3). The expression of KCa2.1 was unchanged in GK atrium compared with control atrium. After 12 weeks of metformin treatment, the expression of $\mathrm{KCa} 2$. 2 was markedly up-regulated while the expression of $\mathrm{KCa} 2.3$ was dramatically down-regulated in the atrium as compared to the untreated GK group $(P<0.01)$.

Next, the mRNA expression levels of the SK channels were measured by real-time PCR. Consistent with the protein levels, the expression of KCNN2 was downregulated and the expression of $K C N N 3$ was up-regulated in the diabetic atrium as compared with control atrium (Fig. 3c, $P<0.01$; Additional file 1: Table S4). Moreover, metformin treatment significantly increased KCNN2 expression and decreased $K C N N 3$ expression in the atrium of GK rats $(P<0.05)$. These results indicated that the expression pattern of the SK channels in rat atrial myocytes was altered under diabetic conditions and metformin abolished diabetes-induced changes in the expression of atrial SK channels.

\section{SK current decline in diabetic atrial myocytes was reversed by metformin}

The electrophysiological changes in the isolated atrial myocytes of GK rats were assessed by patch clamp. Fig. 4a shows the representative tracings of SK currents in the atrial myocytes from three groups of rats. Compared with control atrial myocytes, atrial myocytes from GK rats displayed a significantly reduced intensity of SK current $(P<0.001)$. By contrast, the SK current was markedly increased in the atrial myocytes from metformin-treated GK rats as compared with the untreated ones (Fig. 4b; Additional file 1: Table S5). In addition, the current-voltage relationship of SK currents was distorted in diabetic atrial myocytes, and it was corrected in the atrial myocytes of GK rats with metformin treatment (Fig. 4c; Additional file 1: Table S6).

\section{Metformin minimized diabetes-induced lengthening of APD in atrial myocytes}

Next, we measured action potential in the isolated atrial myocytes from three groups of rats. Before exposure to apamin, RP and APA were similar among the three groups; $\mathrm{APD}_{50}$ and $\mathrm{APD}_{90}$ were significantly prolonged 


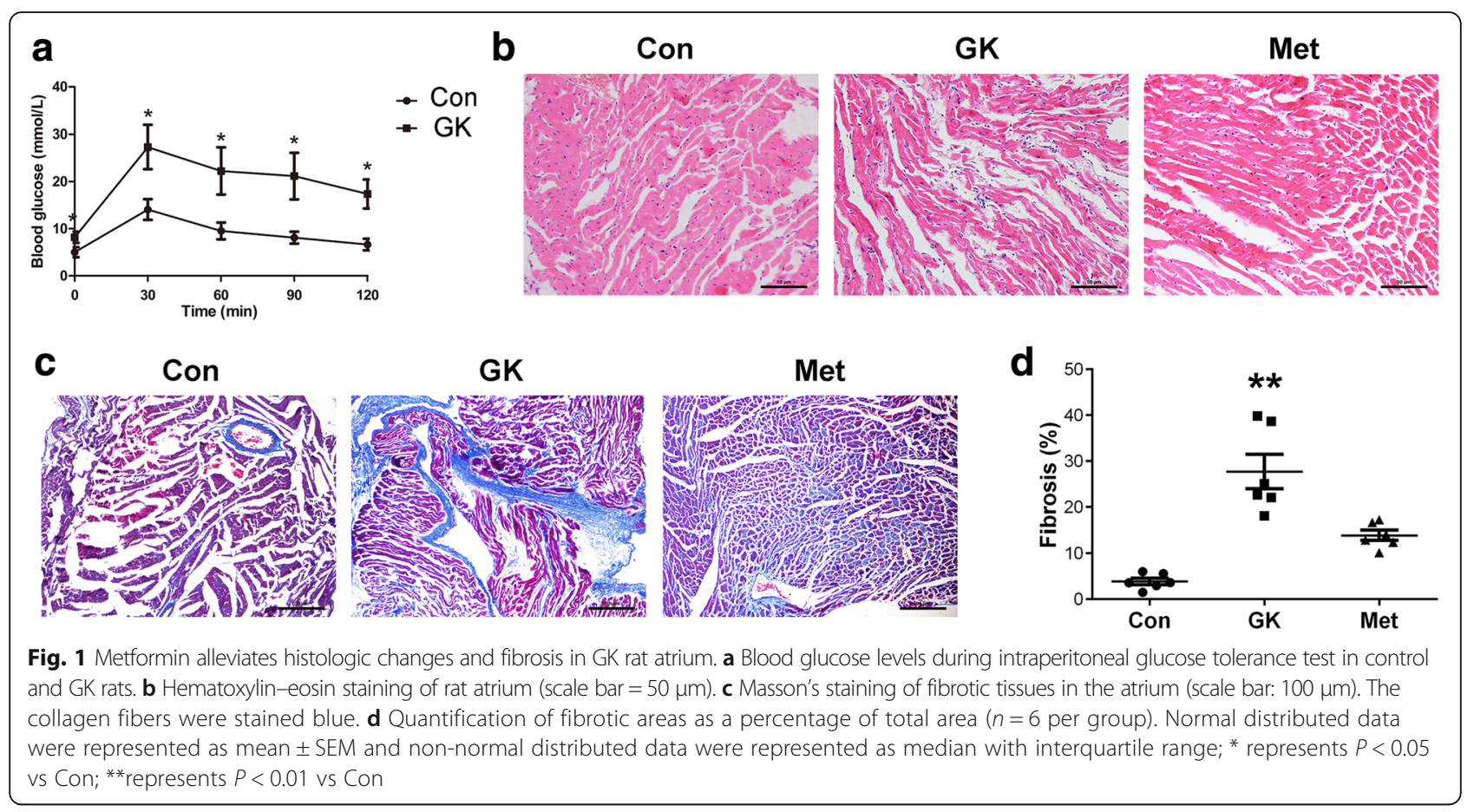

in the GK group $(P<0.01)$, and such lengthening of APD was markedly reduced after metformin treatment (Fig. 5a; Additional file 1: Table S7). Upon exposure to apamin, a specific SK channel blocker, $\mathrm{APD}_{50}$ and $\mathrm{APD}_{90}$ increased by 25.75 and $26.44 \%$, respectively, in the Con group, and by 6.40 and $11.99 \%$, respectively, in the Met group. However, SK channel blockade had no significant effect on APD in the GK group (Fig. 5b and c; Additional file 1: Table S8). These data implied that the function of the SK channels was impaired in the atrial myocytes of diabetic GK rats, and that the function of atrial SK channels was partially restored after metformin treatment.

\section{Discussion}

Our study demonstrates for the first time that the expression and current intensity of SK channels are altered in the atrial myocytes of diabetic GK rats, which leads to the prolongation of APD. Of course, the changes in APD may result from remodeling of multiple ion channels involving transient outward current and calcium current. By specifically blocking the SK channels with apamin, we found that the atrial myocytes from GK

Table 2 Rat body weight and fasting blood glucose level at the end of 12-week metformin treatment

\begin{tabular}{llll}
\hline & Con & GK & Met \\
\hline Weight (g) & $519.33 \pm 9.64$ & $461.92 \pm 4.53^{*}$ & $420.25 \pm 5.28^{* \#}$ \\
FBG $(\mathrm{mM})$ & $4.31 \pm 0.03$ & $13.77 \pm 0.16^{*}$ & $9.45 \pm 0.05$ \\
\hline
\end{tabular}

FBG fasting blood glucose, $n=6$ per group

${ }^{*} P<0.01$ vs Con; ${ }^{*} P<0.01$ vs GK rats did not respond to apamin-induced APD prolongation, indicating that the function of the SK channels was impaired in the atrial myocytes of this T2DM model rat. Consistent with our findings, down-regulation of atrial SK channels in type 1 diabetic mice is associated with a decreased current intensity [20]. In our study, KCa2.2 is the only down-regulated SK channel in diabetic atrium, and it is likely to be responsible for the reduced SK current and prolonged APD in atrial myocytes under diabetic conditions. On the other hand, by taking advantage of a KCNN3 transgenic mouse model, Zhang et al. demonstrate that overexpression of $\mathrm{KCa} 2.3$ results in significant shortening of APD in atrial myocytes and increased susceptibility to atrial fibrillation [21]. Thus, elevated expression of $\mathrm{KCa} 2.3$ is a risk factor of atrial fibrillation. Here, the expression of $\mathrm{KCa} 2.3$ was significantly increased in the atrium of GK rats, suggesting that it may play a role in diabetes-induced arrhythmia.

The relation between ion channel activity and cardiac electrical property is complicated. Clinical data indicate an increased risk of atrial fibrillation in patients with diabetes [6]. In this study, KCa2.2 was down-regulated while $\mathrm{KCa} 2.3$ was up-regulated in the diabetic atrium, implying the potential involvement of the SK channel alteration in the development of arrhythmia or atrial fibrillation. It is reported that down-regulation of $\mathrm{KCa} 2$. 1 and $\mathrm{KCa} 2.2$ is detected along the progression of atrial fibrillation in human [22]. For these differential expression patterns of SK channels, we assume that the expression of SK channels changes with the development of arrhythmia, and the expression pattern depends on the 

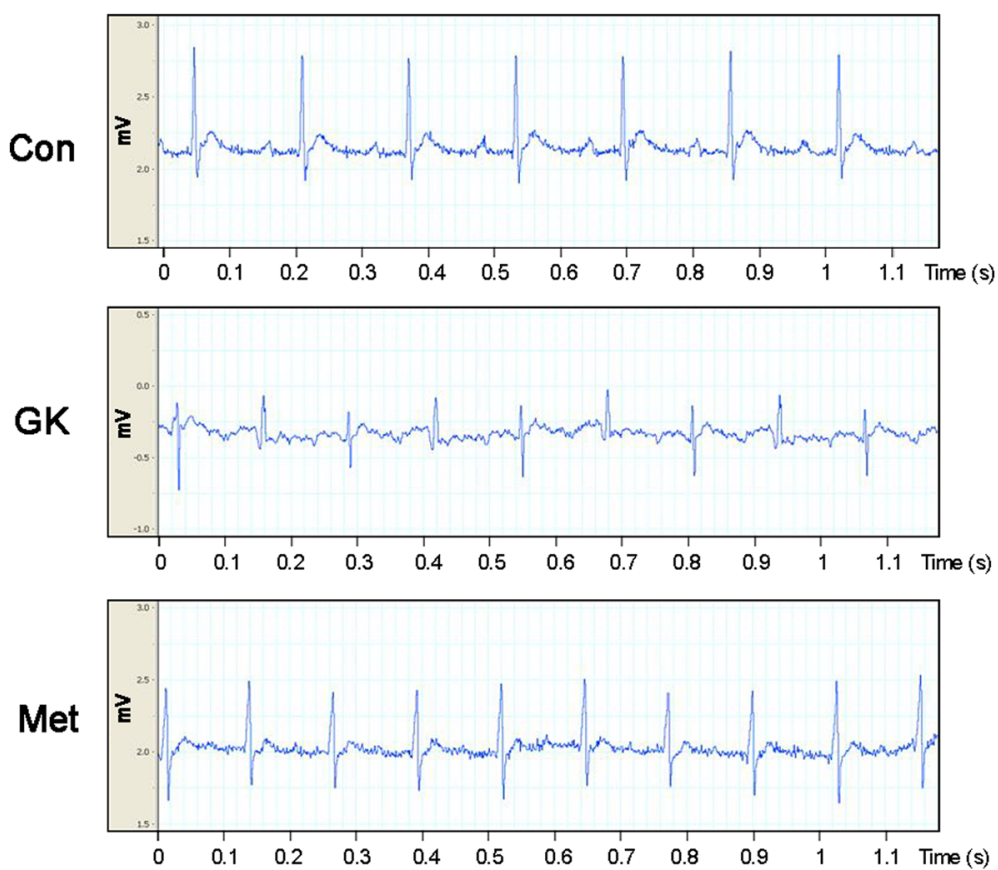

Fig. 2 ECG of control, GK and metformin-treated GK rats. GK rats displayed accelerated heart rate, junctional rhythm, separation of $P$ and QRS waves, irregular $P$ waves and partial blockade of electrical conduction, whereas metformin-treated GK rats showed sinus rhythm and relatively regular $P$ waves

stage of the disease. In addition, blockade of SK channels in various cardiac arrhythmia models has been shown to be both anti-arrhythmic [23] and pro-arrhythmic [7, 24], and both shortening and lengthening of action potential will lead to arrhythmia. Our results showed downregulation of $\mathrm{KCa} 2.2$ and up-regulation of $\mathrm{KCa} 2.3$ were associated with prolonged APD in atrial myocytes and arrhythmia in diabetic rats, yet the relationship between each single SK channel and diabeticassociated arrhythmia need to be addressed in the future. Furthermore, our data suggests that diabetic conditions affected SK channel expression at the mRNA level. Therefore, it is important to identify the upstream transcription factors that regulate the

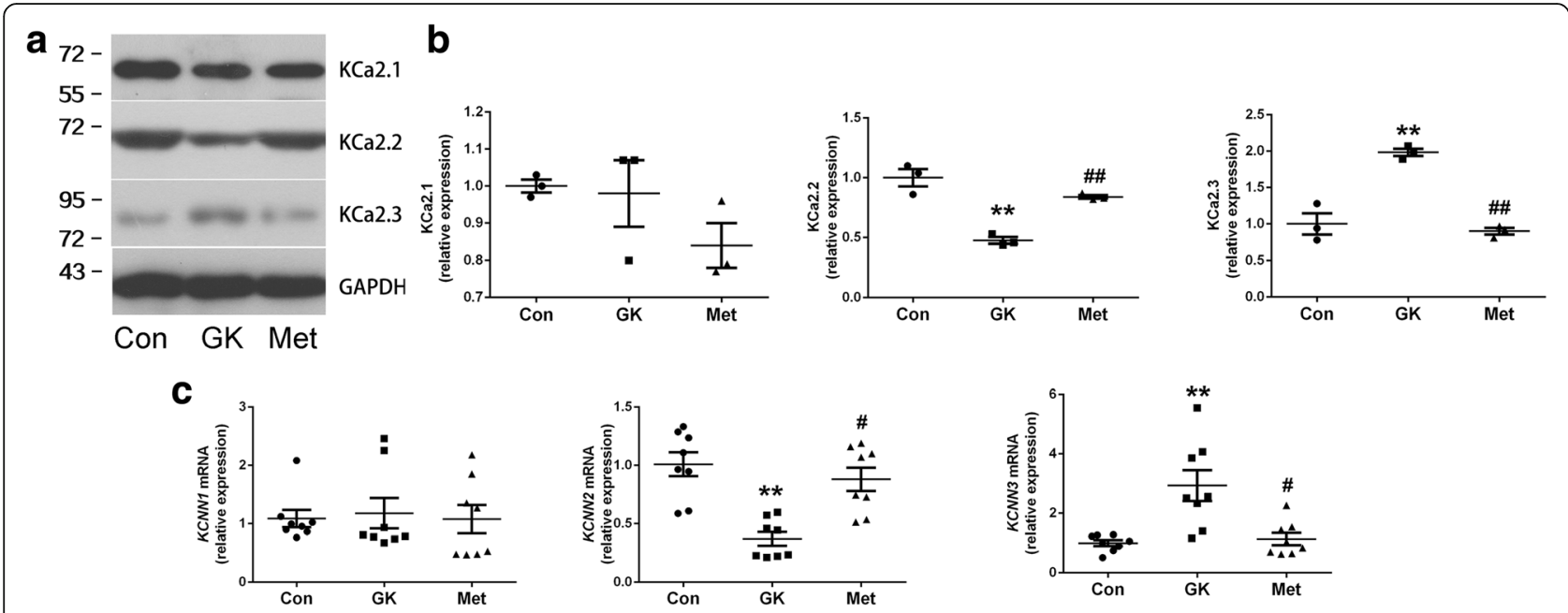

Fig. 3 Expression of KCa2.1, KCa2.2 and KCa2.3 in the atrial tissue of control rats and GK rats with or without metformin treatment. a Representative Western blots and $\mathbf{b}$ relative levels of KCa2.1, KCa2.2 and KCa2.3 in the atrial tissues of three groups of rats ( $n=3$ per group). c Relative levels of KCNN1, KCNN2 and KCNN3 mRNAs in the atrial tissues of three groups of rats ( $n=8$ per group). Non-normal distributed data were represented as median with interquartile range; ** represents $P<0.01$ vs Con; ${ }^{\#}$ represents $P<0.05$ vs GK; \#\# represents $P<0.01$ vs GK 


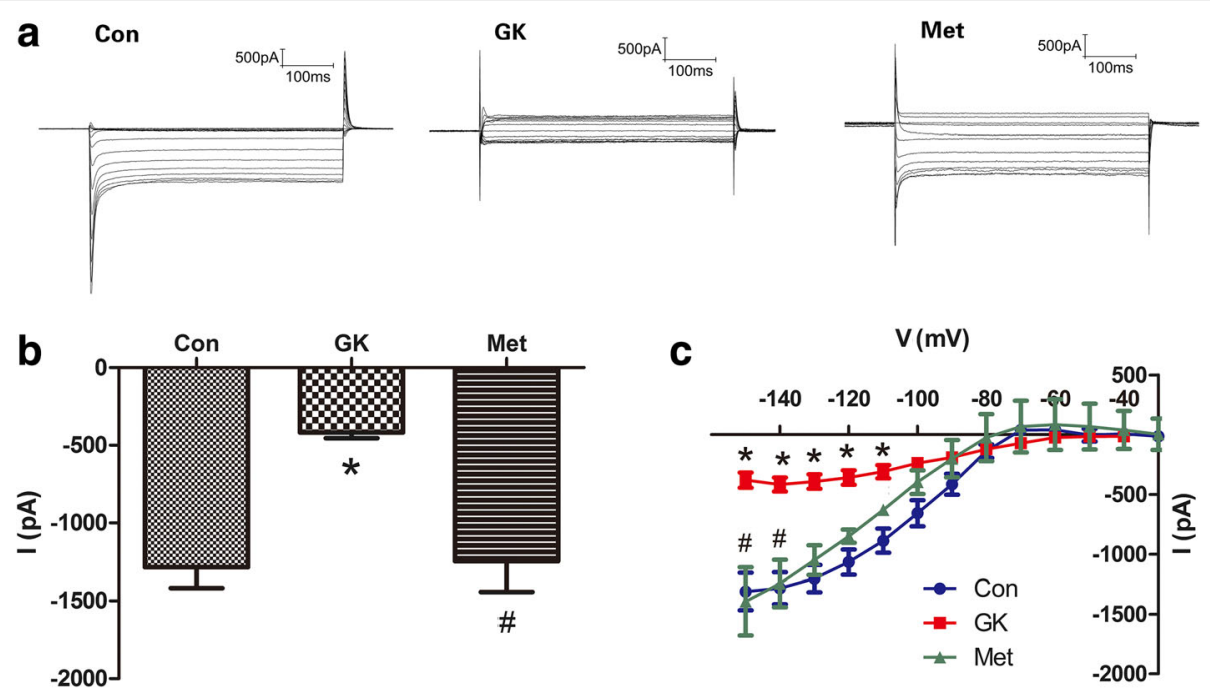

Fig. 4 SK current recordings in atrial myocytes from control, GK and metformin-treated GK rats. a Representative tracings of SK currents. b Mean SK current intensity at $-140 \mathrm{mV}$. c Current-voltage relation of SK currents at voltages ranging from -150 to $-20 \mathrm{mV}$. Results are mean \pm SEM $(n=11-12$ cells from three rats in each group); ${ }^{*}$ represents $P<0.05$ vs Con; ${ }^{\#}$ represents $P<0.05$ vs GK

expression of SK channels and microRNAs that interfere with the stability of SK channel transcripts $[25,26]$, and these factors may become potential targets for the intervention of the pathologic changes (such as atrial arrhythmia) in T2DM.
Metabolic stress, inflammation and oxidative stress may affect cardiac functionality and contribute to arrhythmia, and alterations of ion channel activity are implicated in these pathological processes [27]. In the patients affected by outflow tract premature ventricular

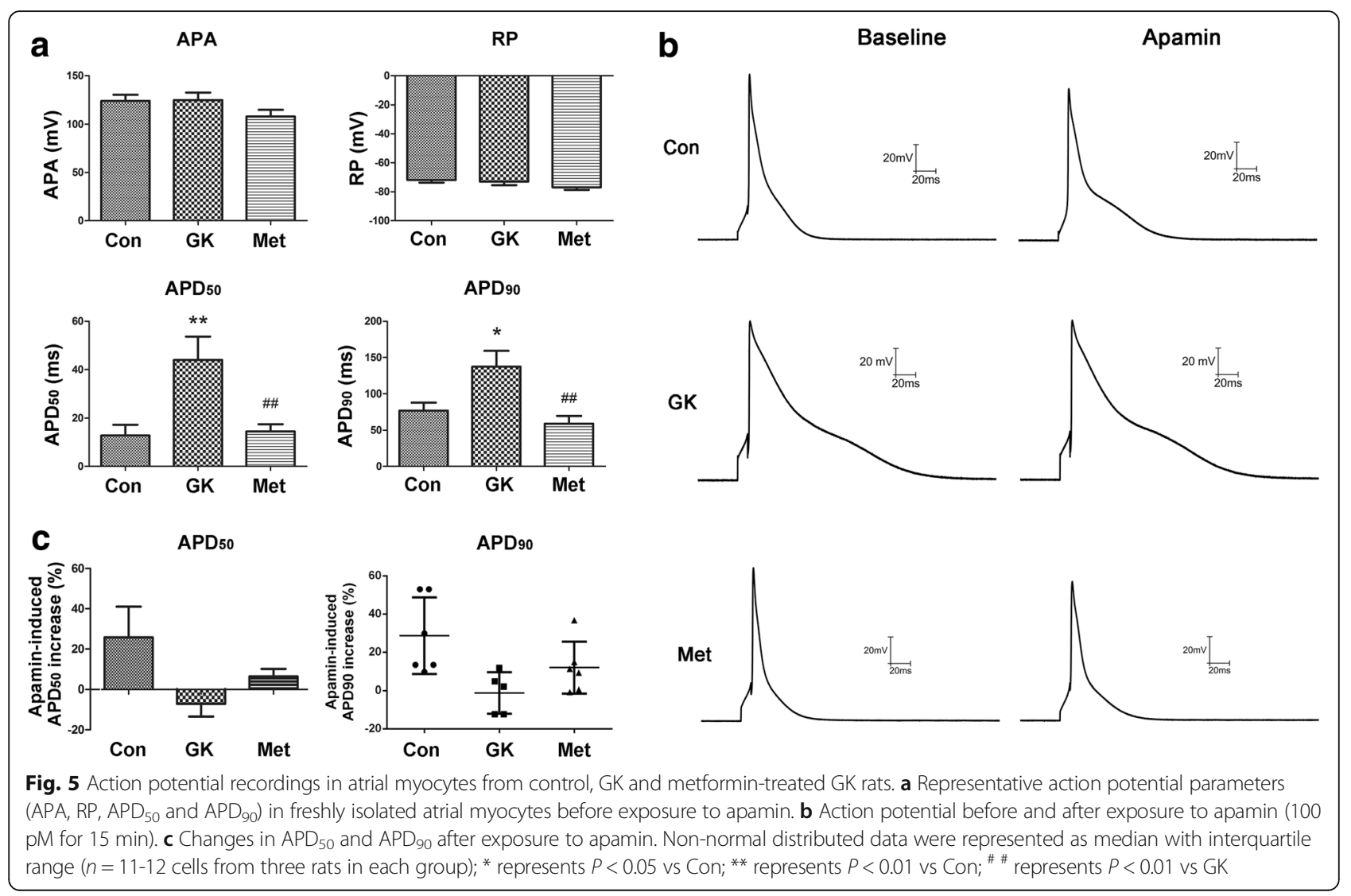


contractions, a higher recurrence rate after catheter ablation was noticed in those with metabolic syndrome [28]. It is mostly likely that alterations of ion channels in the cardiomyocytes affect the long-term post-ablation prognosis. Here we showed that myocardial expression of SK channels was altered under diabetic conditions. Therefore, in addition to the treatment of arrhythmia in T2DM patients, such as cardiac resynchronization $[29,30]$, correction of ion channel expression and activity should be considered for the maintenance of cardiac functionality and prevention of recurrence.

In the present study, the anti-arrhythmic effect of metformin was analyzed. Metformin treatment alleviated diabetes-induced atrial remodeling, fibrosis and arrhythmia in GK rats. Moreover, metformin treatment reversed the changes in the expression of SK channels and preserved SK channel function in the atrial myocytes of diabetic rats. Our findings support the beneficial effect of metformin on cardiac electrophysiology under diabetic conditions. Therefore, this anti-diabetic agent not only stabilizes blood glucose in patients with T2DM, but may also provide protection to the heart and prevent diabetic arrhythmia.

\section{Conclusions}

Our study shows that altered expression and function of SK channels altered in atrial myocytes is associated with arrhythmia in a rat model of T2DM. Moreover, metformin treatment may alleviate diabetic arrhythmia by maintaining the expression and function of atrial SK channels.

\section{Additional file}

Additional file 1: Table S1. Raw data of blood glucose level. Table S2. Raw data of fibrotic area. Table S3. Raw data of western blotting detection of the expressions of KCa2.1, KCa2.2 and KCa2.3. Table S4. Raw data of real-time PCR detection of the expressions of KCNN1, KCNN2, and KCNN3. Table S5. Raw data of SK current intensities at - $140 \mathrm{mV}$. Table S6. Raw data of currentvoltage relation of SK currents at voltages ranging from -150 to $-20 \mathrm{mV}$. Table S7. Raw data of action potential parameters. Table S8. Raw data of action potential increase before and after exposure to apamin. (DOC $178 \mathrm{~kb})$

\section{Abbreviations}

APA: Action potential amplitude; APD: Action potential duration; GK: GotoKakizaki; Met: Metformin; RP: Resting potential; SK channel: Small conductance calcium-activated potassium channel; T2DM: Type 2 diabetes mellitus

\section{Availability of data and material}

All data generated or analyzed during this study are included in this article.

\section{Funding}

This study was supported by grants from the Natural Science Foundation of Ministry of Science and Technology, Liaoning Province [No. 201602839] and the Health and Family Planning Commission of Liaoning Province [No. LNCCC-D10-2015]. The funding bodies had no role in design of the study, collection, analysis and interpretation of data, or manuscript preparation.

\section{Authors' contributions}

$X \mathrm{~L}$ conceived the study. XF and YP conducted the animal experiment and cardiac histology. QC and BL carried out PCR and Western blot analyses. XF, $\mathrm{SW}, \mathrm{HD}$ and ND isolated atrial myocytes and performed the patch clamp assay. $\mathrm{XF}$ and $\mathrm{XL}$ analyzed the data and prepared the manuscript. All authors read and approved the final manuscript.

\section{Ethics approval}

The experiment protocol conformed to the Guide for the Care and Use of Laboratory Animals by National Institutes of Health (NIH Publication No. 80-23) and approved by the Ethics Committee of Shengjing Hospital.

Competing interests

The authors declare that they have no competing interests.

\section{Publisher's Note}

Springer Nature remains neutral with regard to jurisdictional claims in published maps and institutional affiliations.

\section{Author details}

${ }^{1}$ Department of Cardiology, Shengjing Hospital of China Medical University, Shenyang 110004, People's Republic of China. ${ }^{2}$ Department of Cardiology, The People's Hospital of Liaoning Province, Shenyang 110016, People's Republic of China.

Received: 21 December 2017 Accepted: 4 April 2018

Published online: 10 April 2018

\section{References}

1. International Diabetes Federation. IDF Diabetes Atlas. 7th ed. Brussels: International Diabetes Federation; 2015.

2. Saeed A, Ballantyne CM. Assessing cardiovascular risk and testing in type 2 diabetes. Curr Cardiol Rep. 2017;19(3):19.

3. Barbero U, D'Ascenzo F, Nijhoff F, Moretti C, Biondi-Zoccai G, Mennuni M, Capodanno D, Lococo M, Lipinski MJ, Gaita F. Assessing risk in patients with stable coronary disease: when should we intensify care and follow-up? Results from a meta-analysis of observational studies of the COURAGE and FAME era. Scientifica (Cairo). 2016;2016:3769152.

4. Yazici M, Ozdemir K, Altunkeser BB, Kayrak M, Duzenli MA, Vatankulu MA, Soylu A, Ulgen MS. The effect of diabetes mellitus on the P-wave dispersion. Circ J. 2007;71(6):880-3.

5. Pfister R, Cairns R, Erdmann E, Schneider CA, Investigators PR. Prognostic impact of electrocardiographic signs in patients with type 2 diabetes and cardiovascular disease: results from the PROactive study. Diabet Med. 2011; 28(10):1206-12.

6. Huxley RR, Filion KB, Konety S, Alonso A. Meta-analysis of cohort and casecontrol studies of type 2 diabetes mellitus and risk of atrial fibrillation. Am J Cardiol. 2011;108(1):56-62.

7. Skibsbye L, Poulet C, Diness JG, Bentzen BH, Yuan L, Kappert U, Matschke K Wettwer E, Ravens U, Grunnet M, et al. Small-conductance calcium-activated potassium (SK) channels contribute to action potential repolarization in human atria. Cardiovasc Res. 2014;103(1):156-67.

8. Tuteja D, Xu D, Timofeyev V, Lu L, Sharma D, Zhang Z, Xu Y, Nie L, Vazquez $A E$, Young JN, et al. Differential expression of small-conductance Ca2+ -activated K+ channels SK1, SK2, and SK3 in mouse atrial and ventricular myocytes. Am J Physiol Heart Circ Physiol. 2005;289(6):H2714-23.

9. Ellinor PT, Lunetta KL, Glazer NL, Pfeufer A, Alonso A, Chung MK, Sinner MF, de Bakker PI, Mueller M, Lubitz SA, et al. Common variants in KCNN3 are associated with lone atrial fibrillation. Nat Genet. 2010;42(3):240-4.

10. Qi $X Y$, Diness JG, Brundel BJ, Zhou XB, Naud P, Wu CT, Huang H, Harada M, Aflaki M, Dobrev D, et al. Role of small-conductance calcium-activated potassium channels in atrial electrophysiology and fibrillation in the dog. Circulation. 2014;129(4):430-40.

11. Bennett WL, Maruthur NM, Singh S, Segal JB, Wilson LM, Chatterjee R, Marinopoulos SS, Puhan MA, Ranasinghe P, Block L, et al. Comparative effectiveness and safety of medications for type 2 diabetes: an update including new drugs and 2-drug combinations. Ann Intern Med. 2011; 154(9):602-13.

12. Chang SH, Wu LS, Chiou MJ, Liu JR, Yu KH, Kuo CF, Wen MS, Chen WJ, Yeh $\mathrm{YH}$, See LC. Association of metformin with lower atrial fibrillation risk among patients with type 2 diabetes mellitus: a population-based dynamic cohort and in vitro studies. Cardiovasc Diabetol. 2014;13:123.

13. Ashwini S, Bobby Z, Joseph M. Mild hypothyroidism improves glucose tolerance in experimental type 2 diabetes. Chem Biol Interact. 2015;235:47-55. 
14. Zhao LM, Wang Y, Yang Y, Guo R, Wang NP, Deng XL. Metformin restores intermediate-conductance calcium-activated $\mathrm{K}(+)$ channel- and smallconductance calcium-activated $\mathrm{K}(+)$ channel-mediated vasodilatation impaired by advanced glycation end products in rat mesenteric artery. Mol Pharmacol. 2014;86(5):580-91.

15. Abdelsaid M, Kaczmarek J, Coucha M, Ergul A. Dual endothelin receptor antagonism with bosentan reverses established vascular remodeling and dysfunctional angiogenesis in diabetic rats: relevance to glycemic control. Life Sci. 2014;118(2):268-73.

16. Chen J, Li Q, Dong R, Gao H, Peng H, Wu Y. The effect of the Ras homolog gene family (rho), member a/rho associated coiled-coil forming protein kinase pathway in atrial fibrosis of type 2 diabetes in rats. Exp Ther Med. 2014:8(3):836-40

17. Lu T, Zhang DM, Wang XL, He T, Wang RX, Chai Q, Katusic ZS, Lee HC. Regulation of coronary arterial BK channels by caveolae-mediated angiotensin II signaling in diabetes mellitus. Circ Res. 2010;106(6):1164-73.

18. Lee HC, Lu T, Weintraub NL, Van Rollins M, Spector AA, Shibata EF. Effects of epoxyeicosatrienoic acids on the cardiac sodium channels in isolated rat ventricular myocytes. J Physiol. 1999;519(Pt 1):153-68.

19. Li N, Timofeyev V, Tuteja D, Xu D, Lu L, Zhang Q, Zhang Z, Singapuri A, Albert TR, Rajagopal AV, et al. Ablation of a Ca2+-activated K+ channel (SK2 channel) results in action potential prolongation in atrial myocytes and atrial fibrillation. J Physiol. 2009;587(Pt 5):1087-100.

20. Yi F, Ling TY, LU T, Wang XL, Li J, Claycomb WC, Shen WK, Lee HC. Downregulation of the small conductance calcium-activated potassium channels in diabetic mouse atria. J Biol Chem. 2015;290(11):7016-26.

21. Zhang XD, Timofeyev V, Li N, Myers RE, Zhang DM, Singapuri A, Lau VC, Bond CT, Adelman J, Lieu DK, et al. Critical roles of a small conductance $\mathrm{ca}(2)(+)$-activated $\mathrm{K}(+)$ channel (SK3) in the repolarization process of atrial myocytes. Cardiovasc Res. 2014;101(2):317-25.

22. Yu T, Deng C, Wu R, Guo H, Zheng S, Yu X, Shan Z, Kuang S, Lin Q. Decreased expression of small-conductance Ca2+-activated $\mathrm{K}+$ channels SK1 and SK2 in human chronic atrial fibrillation. Life Sci. 2012;90(5-6):219-27.

23. Skibsbye L, Diness JG, Sorensen US, Hansen RS, Grunnet M. The duration of pacing-induced atrial fibrillation is reduced in vivo by inhibition of small conductance ca(2+)-activated K(+) channels. J Cardiovasc Pharmacol. 2011; 57(6):672-81.

24. Hsueh CH, Chang PC, Hsieh YC, Reher T, Chen PS, Lin SF. Proarrhythmic effect of blocking the small conductance calcium activated potassium channel in isolated canine left atrium. Heart Rhythm. 2013;10(6):891-8.

25. Kye MJ, Spiess J, Blank T. Transcriptional regulation of intronic calciumactivated potassium channel SK2 promoters by nuclear factor-kappa B and glucocorticoids. Mol Cell Biochem. 2007;300(1-2):9-17.

26. Ling TY, Wang XL, Chai Q, Lau TW, Koestler CM, Park SJ, Daly RC, Greason KL, Jen J, Wu LQ, et al. Regulation of the SK3 channel by microRNA-499potential role in atrial fibrillation. Heart Rhythm. 2013;10(7):1001-9.

27. Jeong EM, Liu M, Sturdy M, Gao G, Varghese ST, Sovari AA, Dudley SC Jr. Metabolic stress, reactive oxygen species, and arrhythmia. J Mol Cell Cardiol. 2012;52(2):454-63.

28. Sardu C, Carreras G, Katsanos S, Kamperidis V, Pace MC, Passavanti MB, Fava I, Paolisso P, Pieretti G, Nicoletti GF, et al. Metabolic syndrome is associated with a poor outcome in patients affected by outflow tract premature ventricular contractions treated by catheter ablation. BMC Cardiovasc Disord. 2014;14:176.

29. Sardu C, Marfella R, Santulli G. Impact of diabetes mellitus on the clinical response to cardiac resynchronization therapy in elderly people. J Cardiovasc Transl Res. 2014;7(3):362-8.

30. Sardu C, Barbieri M, Santamaria M, Giordano V, Sacra C, Paolisso P, Spirito A, Marfella R, Paolisso G, Rizzo MR. Multipolar pacing by cardiac resynchronization therapy with a defibrillators treatment in type 2 diabetes mellitus failing heart patients: impact on responders rate, and clinical outcomes. Cardiovasc Diabetol. 2017;16(1):75.

\section{Ready to submit your research? Choose BMC and benefit from:}

- fast, convenient online submission

- thorough peer review by experienced researchers in your field

- rapid publication on acceptance

- support for research data, including large and complex data types

- gold Open Access which fosters wider collaboration and increased citations

- maximum visibility for your research: over $100 \mathrm{M}$ website views per year

At BMC, research is always in progress.

Learn more biomedcentral.com/submissions 